\title{
Aus den Verhandlungen des FMH-Zentralvorstands
}

St. An seiner Sitzung vom 19. März 2003 behandelte der FMH-Zentralvorstand unter anderem folgende Geschäfte.

\section{Strukturreform/Standesrecht}

\section{Totalrevision des Opferhilfegesetzes} (OHG), Vorentwurf der Expertenkommission; Vernehmlassung

Der Zentralvorstand genehmigt die Vernehmlassungsantwort, die sich auf medizinische Aspekte beschränkt, zuhanden des Bundesamtes für Justiz. Darin spricht er sich u.a. für die Beibehaltung von Genugtuungszahlungen sowie die Beibehaltung einer gesetzlichen Obergrenze aus. Das Opfer soll zudem eine höhere Genugtuung erhalten können als die Angehörigen. Der ZV unterstützt ausserdem die Melderechte gegenüber Vormundschafts- und Strafverfolgungsbehörden, lehnt aber eine Meldepflicht $a b$, da diese den Zugang zur Opferhilfeberatung erschweren würde. Der ZV unterstützt die Einrichtung einer 24-Stunden-Hotline zur Beratung der Opfer von Menschenhandel, welche auch als konsiliarische Anlaufstelle für die Ärztinnen und Ärzte hilfreich sein könnte, die oft die Opfer häuslicher Gewalt als erste behandeln.

\section{2. Änderung von zwei Verordnungen betreffend Strassenfahrzeuge; Vernehmlassung}

Auch in der Schweiz soll in Übereinstimmung mit den Anforderungen der entsprechenden EURichtlinie die Ausrüstungspflicht mit Geschwindigkeitsbegrenzern auf alle Motorwagen zum Personentransport mit mehr als neun Plätzen und auf Motorwagen zum Sachentransport mit einem Gesamtgewicht von mehr als 3,5 t ausgedehnt werden. Ausserdem sollen die Abgasvorschriften für Motorräder, Kleinmotorfahrzeuge sowie dreirädrige Motorfahrzeuge verschärft und der Schadstoffausstoss weiter vermindert werden.

Der Zentralvorstand stimmt diesen in der Vernehmlassung vorgeschlagenen Änderungen $\mathrm{zu}$.

\section{Qualitätssicherung}

Arbeitsgruppe Mammographiescreening: Beurteilung verschiedener Modelle

Die Arbeitsgruppe, in der auch die FMH vertreten ist, muss der ELK (Eidgenössischen Leistungskommission) Vorschläge unterbreiten, wie die heute unbefriedigende Situation verbessert werden könnte. Die Arbeitsgruppe hat vier Modelle erarbeitet, um eine Lösung für das Problem der ungenügenden Qualität des opportunistischen Mammographiescreenings zu finden.

Das erste Modell basiert auf dem Status quo, d.h. der aktuellen Situation in den Kantonen Waadt, Genf und Wallis; dieses Modell stützt sich $\mathrm{ab}$ auf die europäischen Guidelines. Modell zwei (Szenario light) könnte für Frauen geschaffen werden, die nicht in einem der erwähnten drei Kantone leben; die Frauen erhielten, nach einer Information durch die Krebsliga, alle zwei Jahre einen Gutschein für eine Mammographie (10\% Selbstbehalt). In diesem Modell stellt sich das Problem der Akkreditierung der Radiologen. Modell drei sieht eine Akkreditierung / Zertifizierung der radiologischen Praxen vor; diese müssten eine jährliche Prüfung ablegen. Hier taucht etwa die Frage auf, wie sich die Rezertifizierung gestalten würde. Modell vier schliesslich sähe ein Weiterbestehen des heutigen Zustands vor.

Der ZV erinnert daran, dass er von Anfang an gefordert hat, dass für alle Frauen das gleiche gelten sollte. Die Qualität des Mammographiescreenings setzt vor allem bei den Durchführenden an. Im Rahmen der Weiter- und Fortbildung ist dies sicherzustellen; der Bund hat die Möglichkeit, dies bei der Weiterbildung durchzusetzen, Sache der Radiologen ist es, zu bestimmen, wie dies gelöst werden kann. Als Beispiel könnte die für den Schwangerschaftsultraschall gefundene Lösung dienen. Für den Zentralvorstand jedenfalls entspricht keines der von der Arbeitsgruppe erarbeiteten vier Modelle der Idealvorstellung. 


\section{Organisatorisches}

\section{Jahresrechnung 2002 und Budget 2004 der $\mathrm{FMH}$}

Jahresrechnung 2002 sowie das Budget 2004 werden im Beisein der Finanzkommission besprochen.

Die Jahresrechnung weist einen Überschuss von rund einer Million Franken aus. Dieser Überschuss ist nur halb so gross, wie er eigentlich hätte sein sollen, um die knappe Million Defizit der Abteilung Aus-, Weiter- und Fortbildung (AWF) zu decken und die 1,2 Million Franken Solidaritätsbeitrag aus den Mitteln der FMH an die AWF zu bezahlen. Das Defizit der Abteilung AWF rührt daher, dass man bei der Erstellung des Budgets im April 2001 davon ausgegangen war, dass ab Januar 2002 nur noch eidgenössische Titel für Fr. 4000.- ausgestellt würden. Nachdem die Bilateralen Verträge aber erst auf den 1. Juni 2002 in Kraft getreten sind, konnten die meisten Mitglieder ihre Titel noch zum alten Preis erwerben.

Das Gesamtbudget der FMH für 2004 ist quasi ausgeglichen; die Finanzplanung des Costcenters AWF ergibt für das Budgetjahr einen Verlust, der durch leichte Überschüsse der anderen Abteilungen kompensiert werden kann.

Der Zentralvorstand verabschiedet die Jahresrechnung 2002 und das Budget 2004 zuhanden der ordentlichen Ärztekammer.

\section{Délibérations du Comité central de la FMH}

St. Lors de sa séance du 19 mars 2003, le Comité central de la FMH s'est notamment penché sur les affaires suivantes:

\section{Réformes structurelles/déontologie}

1. Révision totale de la Loi sur l'aide aux victimes d'infractions (LAVI): projet préliminaire de la commission d'experts et procédure de consultation

Le Comité central approuve une prise de position destinée à l'Office fédéral de la justice, qui porte exclusivement sur les aspects médicaux de la question. Il y préconise entre autres de maintenir les indemnités à verser pour tort moral, de même que la limite supérieure fixée par la loi pour ces dernières. De plus, les victimes devraient pouvoir obtenir des indemnités plus élevées que leurs proches. Si le CC soutient le principe du droit d'annoncer les délits à l'autorité de tutelle et à l'autorité pénale, il rejette en revanche celui d'une annonce obligatoire, car celle-ci ferait hésiter les personnes concernées à recourir aux services de conseil. Le CC approuve la mise en place d'une ligne d'assistance téléphonique permanente en vue de conseiller les victimes de la traite des êtres humains. Celle-ci pourrait aussi être consultée par les médecins, qui sont souvent les premiers à traiter les victimes de violences domestiques.

\section{Modification de deux ordonnances concernant les véhicules routiers: procédure de consultation}

Il s'agit d'adapter la législation suisse aux directives de l'UE, en étendant l'obligation de monter des limiteurs de vitesse à tous les véhicules à moteur destinés au transport de personnes et comportant plus de neuf places, ainsi qu'aux véhicules à moteur servant au transport des marchandises et pesant plus de 3,5 tonnes. En outre, les prescriptions relatives aux émissions de polluants provenant des motocycles, des quadricycles légers à moteur et des tricycles à moteur doivent être renforcées. Le Comité central approuve sans modification les propositions soumises à consultation.

\section{Assurance-qualité}

\section{Groupe de travail pour le dépistage} mammographique: évaluation de différents modèles

Ce groupe de travail, dont la FMH fait partie, a pour mission de soumettre à la Commission fédérale des prestations (CFP) des propositions afin d'améliorer le dépistage mammographique. Le groupe a élaboré quatre modèles pour remédier à la qualité insuffisante des dépistages spontanés tels qu'ils sont pratiqués actuellement. 
Le premier modèle se fonde sur le statu quo, à savoir sur la situation qui prévaut actuellement dans les cantons de Vaud, de Genève et du Valais. Il s'appuie sur les directives européennes («guidelines») en la matière. Le deuxième modèle (version allégée) pourrait être appliqué dans le cas des femmes n'habitant pas l'un des trois cantons précités: suite à une information fournie par la Ligue contre le cancer, elles recevraient tous les deux ans un bon pour une mammographie (moyennant une participation de 10\%). Ce modèle soulève la question de l'accréditation des radiologues. Le troisième modèle exige une accréditation ou certification des cabinets de radiologues, qui devraient se soumettre annuellement à un examen. La manière d'organiser le renouvellement de la certification reste à définir. Quant au quatrième modèle, il prévoit le maintien des modes de fonctionnement actuels.

Le CC tient à rappeler que dès le début, il a demandé qu'on mette en place un seul et même système pour toutes les patientes. La qualité du dépistage par mammographie dépend avant tout des compétences de ceux qui l'effectuent. Ces dernières doivent être acquises dans le cadre de la formation postgraduée et continue, laquelle peut être imposée par la Confédération. Quant aux modalités de cette formation, c'est aux radiologues qu'il appartient de les définir. A cet égard, la solution adoptée pour les échographies prénatales pourrait servir d'exemple. Quoi qu'il en soit, le CC estime qu'aucun des quatre modèles développés par le groupe de travail n'offre de solution idéale.

\section{Organisation}

\section{Comptes annuels 2002 et budget 2004 de la FMH}

Le CC débat des comptes et du budget en présence des membres de la Commission des finances.

Les comptes annuels affichent un bénéfice d'environ un million de francs. Celui-ci ne représente que la moitié de la somme qui permettrait, d'une part, de couvrir le déficit de près d'un demi-million de francs du Département de la formation prégraduée, postgraduée et continue (FPPC) et, d'autre part, de verser à celle-ci une contribution de solidarité de 1,2 million de francs provenant des fonds de la FMH. Le déficit de la FPPC est dû au fait que lors de l'établissement du budget en avril 2001, on pensait que seuls des titres fédéraux coûtant Fr. 4000.- seraient encore octroyés à partir de janvier 2002. $\mathrm{Vu}$ l'ajournement au $1^{\text {er }}$ juin de l'entrée en vigueur des accords bilatéraux, la plupart des membres ont encore acquis leur titre à l'ancien prix.

Le budget global de la FMH pour 2004 est pratiquement équilibré. Le déficit prévu par le centre de charges FFPC pour l'année à venir pourra être compensé par de légers excédents de recettes provenant des autres départements.

Le Comité central approuve les comptes annuels 2002 et le budget 2004 et les transmet à la Chambre médicale. 